[5] B. D. Rao and K. V. S. Hari, "Performance analysis of root-music," IEEE Trans. Acoust., Speech, Signal Processing, vol. 37, pp. 1939-1949, Dec. 1989.

[6] _ _ "Performance analysis of ESPRIT and TAM in determining the direction of arrival of plane waves in noise," IEEE Trans. Acoust. Speech, Signal Processing, vol. 37, pp. 1990-1995, Dec. 1989.

[7] M. D. Zoltowski and C. P. Mathews, "Real-time frequency and 2-D angle estimation with sub-Nyquist spatio-temporal sampling," IEEE Trans. Signal Processing, vol. 42, pp. 2781-2794, Oct. 1994.

[8] A. Lee Swindlehurst, B. Ottersten, R. Roy, and T. Kailath, "Multiple invariance ESPRIT," IEEE Trans. Signal Processing, vol. 40, pp. 867-881, Apr. 1992

[9] K. T. Wong and M. D. Zoltowski, "Closed-form multi-dimensional multi-invariance ESPRIT," in Proc. ICASSP, Munich, Germany, Apr. 1997, pp. 3489-3493.

[10] A.-J. van der Veen, P. B. Ober, and E. F. Deprettere, "Azimuth and elevation computation in high resolution DOA estimation," IEEE Trans. Signal Processing, vol. 40, pp. 1828-1832, July 1992.

[11] A.-J. van der Veen and A. Paulraj, "An analytical constant modulus algorithm," IEEE Trans. Signal Processing, vol. 44, pp. 1136-1155, May 1996.

[12] L. de Lathauwer, "Signal processing based on multilinear algebra," Ph.D. dissertation, Katholieke Univ. Leuven, Leuven, Belgium, 1997.

[13] S. Haykin, An Introduction to Analog and Digital Communications. New York: Wiley, 1986.

[14] A. N. Lemma, A.-J. van der Veen, and E. F. Deprettere, "On the multi-resolution ESPRIT algorithm," in Proc. Ninth SP Workshop SSAP, Portland, OR, Sept. 1998.

\section{Analysis of Spatial Smoothing with Uniform Circular Arrays}

\author{
K. Maheswara Reddy and V. U. Reddy
}

\begin{abstract}
In this correspondence, we analyze spatial smoothing with uniform circular arrays (UCA's). In particular, we study the performance of the Root-MUSIC with smoothing in the presence of correlated sources, finite data perturbations, and errors in transformed steering vector that arise due to some approximations made while extending the Root-MUSIC and smoothing to UCA. Expressions are derived for the asymptotic performance of the Root-MUSIC with smoothing applied to the transformed UCA data. An attempt has been made to bring out the impact of both the forward and forward-backward smoothing. We consider UCA's with isotropic as well as directional sensors in our study. Computer simulations are provided to demonstrate the usefulness of the analysis.
\end{abstract}

\section{INTRODUCTION}

Uniform circular arrays (UCA's) are commonly employed when $360^{\circ}$ coverage is required in the plane of the array. Circular arrays are nonuniform linear arrays, and hence, the rooting techniques and preprocessing schemes like spatial smoothing [7] cannot be directly applied to these arrays. In [8], Tewfik and Hong have shown that it is possible to extend the Root-MUSIC to UCA using the phase mode excitation concept. In [2], Mathews and Zoltowski proposed

Manuscript received December 11, 1996; revised November 19, 1998. The associate editor coordinating the review of this paper and approving it for publication was Dr. Gary F. Hatke.

K. M. Reddy is with CASSA, Defence Research and Development Organization, New Thippasandra, Bangalore, India.

V. U. Reddy is with the Department of Electrical Communication Engineering, Indian Institute of Science, Bangalore, India.

Publisher Item Identifier S 1053-587X(99)03679-X. real beamspace MUSIC to UCA that yields reduced computation and better resolution. In [10] and [11], the authors extend spatial smoothing to UCA's.

While extending the rooting techniques to UCA, all the authors assumed that some of the terms in the transformed steering vector of UCA are negligible when the circumferential spacing between the elements is less than half wavelength. These approximations cause errors in the DOA estimates obtained with the Root-MUSIC, even when the number of snapshots tends to infinity, and we analyze the effect of smoothing on these errors in this correspondence. We also extend smoothing to UCA's with directional elements.

\section{BACKGROUND}

Consider a UCA with $L$ identical and omnidirectional sensors. Let $r$ be the radius of the array and $d$ be the circumferential spacing between the elements. Let $\theta$ denote the angle (azimuth angle) measured in the plane containing the elements. We assume for simplicity that the sources are in the same plane as the UCA. The steering vector of the UCA w.r.t. the center of the array can then be expressed as

$$
\begin{gathered}
\mathbf{a}_{c}(\theta)=\left[e^{j \xi \cos \theta}, e^{j \xi \cos (\theta-2 \pi / L)}, \cdots\right. \\
\left.e^{j \xi \cos (\theta-2 \pi(L-1) / L)}\right]^{T}
\end{gathered}
$$

where $\xi=2 \pi r / \lambda, \lambda$ is the wavelength, and $(.)^{T}$ represents the transpose of (.). The weight vector that excites the array with $m$ th phase mode is given by [2] $\mathbf{w}_{m}^{H}=j^{-|m|} / L\left[1, e^{j 2 \pi m / L}, \cdots, e^{j 2 \pi m(L-1) / L}\right]$. The array pattern for the $m$ th phase mode is [1], [2]

$$
\begin{aligned}
f_{m}(\theta)= & \mathbf{w}_{m}^{H} \mathbf{a}_{c}(\theta)=J_{|m|}(\xi) e^{j m \theta} \\
+ & j^{-|m|} \sum_{q=1}^{\infty}\left[j^{g} J_{g}(\xi) e^{-j g \theta}+j^{h} J_{h}(\xi) e^{j h \theta}\right] \\
& \quad-\mathcal{D} \leq m \leq \mathcal{D}
\end{aligned}
$$

where $\mathcal{D}$ is the maximum number of phase modes and given by [2] $\mathcal{D} \simeq\lfloor 2 \pi r / \lambda\rfloor, J_{m}(\xi)$ is the Bessel function of the first kind of order $m, h=L q+m, g=L q-m,(.)^{H}$ represents the complex conjugate transpose of (.), and $\lfloor x\rfloor$ denotes the largest integer less than or equal to $x$. The first term in (2) becomes dominant if $d$ is less than $0.5 \lambda$. In our analysis, we consider $d<0.5 \lambda$ and assume the second term of (2) to be small.

The normalized transformation matrix $\mathbf{F}$ to excite the array patterns corresponding to $(2 \mathcal{D}+1)$ phase modes is given by $\mathbf{F}=$ $\sqrt{L}\left[\mathbf{w}_{-\mathcal{D}}, \cdots, \mathbf{w}_{0}, \cdots, \mathbf{w}_{\mathcal{D}}\right]$. Using this transformation, we express

$$
\mathbf{a}_{t}(\theta)=\mathbf{F}^{H} \mathbf{a}_{c}(\theta)=\mathbf{J}_{\xi} \mathbf{a}(\theta)+\Delta \mathbf{a}(\theta)
$$

where $\mathbf{J}_{\xi}=\sqrt{L} \operatorname{diag}\left[J_{\mathcal{D}}(\xi), \cdots, J_{1}(\xi), J_{0}(\xi), J_{1}(\xi), \cdots, J_{\mathcal{D}}(\xi)\right]$

$$
\mathbf{a}(\theta)=\left[e^{-j \mathcal{D} \theta}, e^{-j(\mathcal{D}-1) \theta}, \cdots, 1, \cdots, e^{j(\mathcal{D}-1) \theta}, e^{j \mathcal{D} \theta}\right]^{T}
$$

and $\Delta \mathbf{a}(\theta)$ is the contribution due to the second term in (2). Note that the vector $\mathbf{a}(\theta)$ has a structure similar to that of the steering vector of a uniform linear array (ULA). We treat $\Delta \mathbf{a}(\theta)$ as the error in the transformed steering vector caused due to approximation.

Assume that $M$ sources are impinging on the UCA and the DOA's of these sources are $\theta_{1}, \theta_{2}, \cdots, \theta_{M}$. The covariance matrix at the output of UCA can be expressed as

$$
\mathbf{R}_{c}=\mathbf{A}_{c} \mathbf{S} \mathbf{A}_{c}^{H}+\sigma^{2} \mathbf{I}
$$


where

S signal covariance matrix;

I identity matrix;

$\mathbf{A}_{c}$ matrix of direction vectors of the UCA.

From (3) and (5), the covariance matrix that we obtain after applying the transformation $\mathbf{F}$ is given by

$$
\mathbf{R}^{t}=\mathbf{F}^{H} \mathbf{R}_{c} \mathbf{F}=\mathbf{J}_{\xi} \mathbf{A} \mathbf{S} \mathbf{A}^{H} \mathbf{J}_{\xi}+\sigma^{2} \mathbf{I}+\mathbf{\Delta} \mathbf{R}
$$

where $\mathbf{A}=\left[\mathbf{a}\left(\theta_{1}\right), \cdots, \mathbf{a}\left(\theta_{M}\right)\right]$, and $\boldsymbol{\Delta} \mathbf{R}=\boldsymbol{\Delta} \mathbf{A} \mathbf{S} \mathbf{A}^{H} \mathbf{J}_{\xi}+$ $\mathbf{J}_{\xi} \mathbf{A} \mathbf{S} \Delta \mathbf{A}^{H}+\boldsymbol{\Delta} \mathbf{A} \mathbf{S} \boldsymbol{\Delta} \mathbf{A}^{H}$ with $\Delta \mathbf{A}=\left[\boldsymbol{\Delta} \mathbf{a}\left(\theta_{1}\right), \cdots, \boldsymbol{\Delta} \mathbf{a}\left(\theta_{M}\right)\right]$. The spatial smoothing can now be extended to UCA by applying the transformation $\mathbf{J}_{\xi}^{-1}$ to the covariance matrix $\mathbf{R}^{t}$, provided the term $\Delta \mathbf{R}$ is negligible. The forward smoothed covariance matrix (with $K$ denoting the number of virtual subarrays) is

$$
\mathbf{R}_{f}^{t}=\frac{1}{K} \sum_{l=1}^{K} \mathbf{Z}_{l}^{T} \mathbf{J}_{\xi}^{-1} \mathbf{R}^{t} \mathbf{J}_{\xi}^{-1} \mathbf{Z}_{l}
$$

where $\mathbf{Z}_{l}=\left[\mathbf{e}_{l}, \mathbf{e}_{l+1}, \cdots, \mathbf{e}_{l+L_{o}-1}\right]$ with $\mathbf{e}_{l}$ denoting the $l$ th column of the identity matrix of size $2 \mathcal{D}+1$, and $L_{0}$ is the virtual subarray dimension with $L_{o}=2 \mathcal{D}-K+2$. Note from (6) and (7) that the noise part of $\mathbf{R}_{f}^{t}$ is diagonal but not of the form $\sigma^{2} \mathbf{I}$. For convenience, we use prewhitening before applying the Root-MUSIC. The forward smoothed covariance matrix after prewhitening is given by

$$
\left(\mathbf{R}_{f}^{t}\right)_{w}=\mathbf{R}_{n w} \mathbf{R}_{f}^{t} \mathbf{R}_{n w}^{H}
$$

where the prewhitening matrix

$$
\mathbf{R}_{n w}=\left[\frac{1}{K} \sum_{l=1}^{K} \mathbf{Z}_{l}^{T} \mathbf{J}_{\xi}^{-1} \mathbf{J}_{\xi}^{-1} \mathbf{Z}_{l}\right]^{-1 / 2} .
$$

\section{ANALYSIS OF ForWARD SMOOTHING WITH UCA}

The steering vector of the ULA is dependent on $\omega=2 \pi d / \lambda \sin \theta$, and hence, the DOA estimation with ULA becomes ambiguous beyond the range of $180^{\circ}$ (from $-90^{\circ}$ to $+90^{\circ}$ ). On the other hand, the vector $\mathbf{a}(\theta)$ is directly dependent on $\theta$, and hence, the DOA estimation with UCA can be carried out unambiguously up to $360^{\circ}$. In addition, the effective correlation between the sources with forward smoothing for UCA is different from that for the ULA. Using the structure of $\mathbf{a}(\theta)$ as given in (4), it can be shown (following the steps in [5]) that the effective correlation coefficient $\left(\rho_{f}\right)$ between the sources is (neglecting $\Delta \mathbf{R}$ )

$$
\left|\rho_{f}\right|=\left|\rho \frac{\sin \left(K\left(\theta_{i}-\theta_{j}\right) / 2\right)}{K \sin \left(\left(\theta_{i}-\theta_{j}\right) / 2\right)}\right|
$$

where $\theta_{i}$ and $\theta_{j}$ are the DOA's of the $i$ th and $j$ th sources, respectively, and $\rho$ is the correlation coefficient before smoothing. Note from (9) that $\rho_{f}$ is not dependent on the individual directions of the sources but is dependent only on their angular separation. If this angular separation is $90^{\circ}$, then $\rho_{f}$ becomes zero for $K=4$. On the other hand, if the separation is $180^{\circ}$, then two subarrays $(K=2)$ are enough to force $\rho_{f}$ to zero. Note also from (9) that $\rho_{f}$ is independent of the spacing between the elements, provided $d$ is much less than $\lambda / 2$. In contrast, when the forward spatial smoothing is applied to ULA, the effective correlation between the sources is dependent on the individual directions of the sources as well as on the spacing between the elements.

We now consider the performance of the Root-MUSIC with forward smoothing applied to the transformed UCA data. Recall the smoothed covariance matrix after prewhitening [see (8)]. Combining this with (6) and (7), we obtain

$$
\left(\mathbf{R}_{f}^{t}\right)_{w}=\mathbf{R}_{\mathbf{F}}+\Delta \mathbf{R}_{\mathbf{F}}
$$

where

$$
\begin{aligned}
\mathbf{R}_{\mathbf{F}} & \equiv \frac{1}{K} \sum_{l=1}^{K} \mathbf{R}_{n w} \mathbf{Z}_{l}^{T} \mathbf{A} \mathbf{S} \mathbf{A}^{H} \mathbf{Z}_{l} \mathbf{R}_{n w}^{H}+\sigma^{2} \mathbf{I} \\
& =\mathbf{R}_{n w} \mathbf{A}_{f} \mathbf{S}_{f} \mathbf{A}_{f}^{H} \mathbf{R}_{n w}^{H}+\sigma^{2} \mathbf{I}
\end{aligned}
$$

and

$$
\begin{aligned}
\Delta \mathbf{R}_{\mathbf{F}} \equiv & \frac{1}{K} \sum_{l=1}^{K} \mathbf{R}_{n w} \mathbf{Z}_{l}^{T} \mathbf{J}_{\xi}^{-1} \boldsymbol{\Delta} \mathbf{R} \mathbf{J}_{\xi}^{-1} \mathbf{Z}_{l} \mathbf{R}_{n w}^{H} \\
= & \frac{1}{K} \sum_{l=1}^{K} \mathbf{R}_{n w} \mathbf{Z}_{l}^{T}\left[\mathbf{J}_{\xi}^{-1} \boldsymbol{\Delta} \mathbf{A} \mathbf{S} \mathbf{A}^{H}+\mathbf{A} \mathbf{S} \boldsymbol{\Delta} \mathbf{A}^{H} \mathbf{J}_{\xi}^{-1}\right] \\
& \cdot \mathbf{Z}_{l} \mathbf{R}_{n w}^{H}
\end{aligned}
$$

where $\mathbf{S}_{f}$ is the smoothed signal covariance matrix, and $\mathbf{A}_{f}$ is the virtual subarray direction matrix. In writing the right-hand side of (12), we assumed $\Delta \mathbf{A}$ to be small and neglected the terms containing more than one $\Delta \mathbf{A}$. Note that $\mathbf{R}_{\mathbf{F}}$ is the smoothed covariance matrix that we would get if $\Delta \mathbf{a}(\theta)$ is zero. In practice, however, this term may be small but nonzero, thereby resulting in errors in the DOA estimates when we apply the Root-MUSIC to $\left(\mathbf{R}_{f}^{t}\right)_{w}$. Here, we analyze the effect of this term (i.e., $\Delta \mathbf{R}_{\mathbf{F}}$ ) on the DOA estimates.

Assuming that the noise at the output of the sensors is complex circularly Gaussian distributed, the mean square error (MSE) in the $i$ th DOA estimate, due to both the finite data perturbations and the error due to approximation (i.e., due to $\Delta \mathbf{R}_{\mathbf{F}}$ ), can be shown to be (following the steps in [4])

$$
\begin{aligned}
E\left[\Delta \theta_{i}^{2}\right]_{f} & =\frac{\boldsymbol{\Gamma}_{\alpha \alpha \beta \beta}+\operatorname{Re}\left(\boldsymbol{\Gamma}_{\alpha \beta \alpha \beta}\right)+\mathbf{2}\left[\operatorname{Re}\left(\alpha^{H} \boldsymbol{\Delta} \mathbf{R}_{\mathbf{F}} \beta\right)\right]^{2}}{\mathbf{2}\left[\mathbf{v}_{f_{1}}^{H}\left(\theta_{i}\right) \mathbf{R}_{n w}^{H} \mathbf{P}_{n} \mathbf{R}_{n w} \mathbf{v}_{f_{1}}\left(\theta_{i}\right)\right]^{2}} \\
\boldsymbol{\Gamma}_{\alpha \alpha \beta \beta} & =\frac{1}{N K^{2}} \sum_{p=1}^{K} \sum_{q=1}^{K} \alpha^{H} \mathbf{R}_{p q} \alpha \beta^{H} \mathbf{R}_{q p} \beta \\
\boldsymbol{\Gamma}_{\alpha \beta \alpha \beta} & =\frac{1}{N K^{2}} \sum_{p=1}^{K} \sum_{q=1}^{K} \alpha^{H} \mathbf{R}_{p q} \beta \alpha^{H} \mathbf{R}_{q p} \beta \\
\alpha & =\mathbf{P}_{n} \mathbf{R}_{n w} \mathbf{v}_{f_{1}}\left(\theta_{i}\right) ; \quad \beta=\left(\mathbf{R}_{\mathbf{F}}\right)_{s}^{\#} \mathbf{R}_{n w} \mathbf{v}_{f}\left(\theta_{i}\right) \\
\left(\mathbf{R}_{\mathbf{F}}\right)_{s} & =\mathbf{R}_{n w} \mathbf{A}_{f} \mathbf{S}_{f} \mathbf{A}_{f}^{H} \mathbf{R}_{n w}^{H}
\end{aligned}
$$

where $\mathbf{R}_{p q}=\mathbf{R}_{q p}^{H}=E\left[\mathbf{y}_{p}(t) \mathbf{y}_{q}^{H}(t)\right], \mathbf{y}_{p}(t)$ is the output vector obtained from the $p$ th virtual subarray after prewhitening, $N$ is the number of snapshots, $\mathbf{P}_{n}$ is the projection matrix onto the noise subspace of $\mathbf{R}_{\mathbf{F}}, \mathbf{v}_{f_{1}}(\theta)$ is the derivative of $\mathbf{v}_{f}(\theta)$ w.r.t. $\theta$ with $\mathbf{v}_{f}(\theta)$ denoting the normalized virtual subarray steering vector, $\operatorname{Re}($. represents the real part of (.), (.) $\#$ denotes the pseudo inverse of (.), and $E($.$) denotes the expectation of (.).$

As the number of snapshots tends to infinity, the MSE in the DOA estimate is only because of the error due to the approximation [c.f. (3)]. This error, which we refer to as the asymptotic error in the DOA estimate, is deterministic and given by

$$
E\left[\Delta \theta_{i}^{2}\right]_{f}=\Delta \theta_{i}^{2}=\frac{\left[\operatorname{Re}\left(\alpha^{H} \boldsymbol{\Delta} \mathbf{R}_{\mathbf{F}} \beta\right)\right]^{2}}{\left[\mathbf{v}_{f_{1}}^{H}\left(\theta_{i}\right) \mathbf{R}_{n w}^{H} \mathbf{P}_{n} \mathbf{R}_{n w} \mathbf{v}_{f_{1}}\left(\theta_{i}\right)\right]^{2}} .
$$

Note that this error increases as $d$ tends to $\lambda / 2$ since $\Delta \mathbf{A}$ becomes larger with increasing value of $d$. Let us first assume that the sources are uncorrelated. Then, the asymptotic error can be shown to be (see [6] for the steps)

$$
\Delta \theta_{i}^{2}=\frac{1}{L_{o}} \frac{\left[\operatorname{Re}\left(\alpha^{H} \mathbf{R}_{n w} \Delta \mathbf{a}_{f}\left(\theta_{i}\right)\right)\right]^{2}}{\left[\mathbf{v}_{f_{1}}^{H}\left(\theta_{i}\right) \mathbf{R}_{n w}^{H} \mathbf{P}_{n} \mathbf{R}_{n w} \mathbf{v}_{f_{1}}\left(\theta_{i}\right)\right]^{2}}
$$

where

$$
\boldsymbol{\Delta} \mathbf{a}_{f}\left(\theta_{i}\right)=\frac{1}{K} \sum_{l=1}^{K} \mathbf{Z}_{l}^{T} \mathbf{J}_{\xi}^{-1} \boldsymbol{\Delta} \mathbf{a}\left(\theta_{i}\right) \mathbf{e}^{-j(l-1) \theta_{i}}
$$




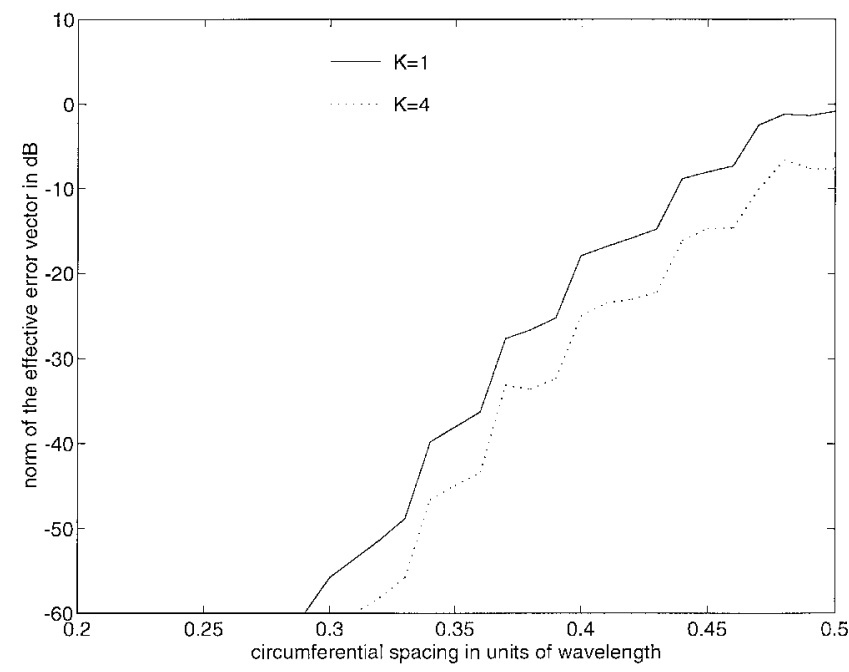

Fig. 1. Norm of the effective error vector in the transformed steering vector of UCA as a function of circumferential spacing $(L=30)$.

which we define as the effective error along the direction of the $i$ th source in the transformed steering vector due to the approximation. Note from (17) that the asymptotic error in the $i$ th DOA estimate is dependent only on the effective error vector $\Delta \mathbf{a}_{f}\left(\theta_{i}\right)$. The norm of this vector can be shown to decrease with smoothing (see [6, App. A] for the steps). Thus, we can expect the smoothing to improve the asymptotic performance. Expression (17), however, holds well only when the sources are uncorrelated. For the case of correlated sources, simplification of (16) leads to lengthy expressions even for a two-source case and, hence, is not considered here.

\section{Forward-Backward Spatial SMOOTHING}

Forward-backward spatial smoothing [9] can also be extended to UCA by applying the transformation $\mathbf{J}_{\xi}^{-1}$ to the covariance matrix $\mathbf{R}^{t}$ [given in (6)], provided $\boldsymbol{\Delta} \mathbf{R}$ is small. The forward-backward smoothed covariance matrix is given by

$$
\mathbf{R}_{f b}^{t}=\frac{1}{2 K} \sum_{l=1}^{K} \mathbf{Z}_{l}^{T}\left[\mathbf{J}_{\xi}^{-1} \mathbf{R}^{t} \mathbf{J}_{\xi}^{-1}+\tilde{\mathbf{I}}\left(\mathbf{J}_{\xi}^{-1} \mathbf{R}^{t} \mathbf{J}_{\xi}^{-1}\right)^{*} \tilde{\mathbf{I}}\right] \mathbf{Z}_{l}
$$

where $\tilde{\mathbf{I}}$ is the exchange matrix, and $(.)^{*}$ denotes complex conjugate of (.). The prewhitening matrix in this case is also given by $\mathbf{R}_{n w}$ (which is defined in Section II) because $\tilde{\mathbf{I}}\left(\mathbf{J}_{\xi}^{-1} \mathbf{J}_{\xi}^{-1}\right)^{*} \tilde{\mathbf{I}}=\mathbf{J}_{\xi}^{-1} \mathbf{J}_{\xi}^{-1}$. In addition, from (4), $\tilde{\mathbf{I}} \mathbf{A}^{*}=\mathbf{A}$. Hence, when the signal covariance matrix $\mathbf{S}$ is real (implying that the signals are uncorrelated or the correlation between the signals is real), $\mathbf{R}_{f b}^{t}$ reduces to $\mathbf{R}_{f}^{t}$. This suggests that the forward-backward smoothing gives the same performance as the forward smoothing when $\mathbf{S}$ is real, which is not so in the case of ULA. When $\mathbf{S}$ is complex, the forward-backward smoothing handles up to $\lfloor 2(2 \mathcal{D}+1) / 3\rfloor$ coherent signals (see [9]) in contrast to the forward smoothing, which handles up to $\lfloor(2 \mathcal{D}+1) / 2\rfloor$ coherent signals only.

Using the structure of $\mathbf{a}(\theta)$ given in (4), it can be shown that the effective correlation coefficient $\left(\rho_{f b}\right)$ between the sources after forward-backward smoothing is given by

$$
\left|\rho_{f b}\right|=|\rho|\left|\frac{\sin \left(K\left(\theta_{i}-\theta_{j}\right) / 2\right) \cos \psi}{K \sin \left(\left(\theta_{i}-\theta_{j}\right) / 2\right)}\right|
$$

where $\rho=|\rho| e^{j \psi}$. Note from (9) and (20) that the effective correlation with forward-backward smoothing is same as that with forward smoothing only, when $\psi$ is zero, i.e., $\rho$ is real. When $\psi$ is
TABLE I

Asymptotic Performance of the Root-MUSIC with SMOothing (APPlicable to Both ForWARD AND ForWARd-BACKWARd, Since $\rho$ is Real) Applied to Transformed UCA Data with ISOTROPIC ELEMENTS FOR UNCORRELATED Two-SOURCE CASE $\left(L=30, D=0.46 \lambda\right.$, DOA's $=0^{\circ}$ AND $7^{\circ}, \rho=0$, SNR $\left.=3 \mathrm{~dB}\right)$

\begin{tabular}{c|c|c}
\hline $\begin{array}{c}\text { Number of } \\
\text { virtual } \\
\text { subarrays, } K\end{array}$ & $\begin{array}{c}\Lambda \text { symptotic performance of the Root-MUSIC } \\
\text { with smoothing }\left(\Delta \theta_{i}^{2} \text { in } \mathrm{deg}^{2}\right)\end{array}$ \\
\cline { 2 - 3 } & From the algorithm & Evaluation of $(17)$ \\
\hline 1 & $0.4852 \times 10^{-1}$ & $0.5359 \times 10^{-1}$ \\
2 & $0.1110 \times 10^{-1}$ & $0.1148 \times 10^{-1}$ \\
3 & $0.2002 \times 10^{-1}$ & $0.2018 \times 10^{-1}$ \\
4 & $0.4491 \times 10^{-2}$ & $0.4684 \times 10^{-2}$ \\
5 & $0.3120 \times 10^{-3}$ & $0.3594 \times 10^{-3}$ \\
6 & $0.5329 \times 10^{-3}$ & $0.5958 \times 10^{-3}$ \\
7 & $0.2543 \times 10^{-2}$ & $0.2624 \times 10^{-2}$ \\
8 & $0.1255 \times 10^{-3}$ & $0.1524 \times 10^{-3}$ \\
9 & $0.2663 \times 10^{-3}$ & $0.3085 \times 10^{-3}$ \\
10 & $0.3612 \times 10^{-3}$ & $0.4167 \times 10^{-3}$ \\
11 & $0.5708 \times 10^{-3}$ & $0.6524 \times 10^{-3}$ \\
12 & $0.1155 \times 10^{-2}$ & $0.1289 \times 10^{-2}$ \\
\multicolumn{2}{|c}{} \\
\hline
\end{tabular}

TABLE II

Asymptotic Performance of the Root-MUSIC with SMOothing (APPlicable to Both ForWARd AND ForWARD-BACKWARD, Since $\rho$ is Real) Applied to Transformed UCA Data with Isotropic Elements For Correlated Two-SOURCE CASE $\left(L=30, d=0.46 \lambda\right.$, DOA's $=0^{\circ}$ AND $\left.7^{\circ}, \rho=0.9, \mathrm{SNR}=3 \mathrm{~dB}\right)$

\begin{tabular}{c|c|c}
\hline $\begin{array}{c}\text { Number of } \\
\text { virtual } \\
\text { subarrays, } K\end{array}$ & $\begin{array}{c}\text { Asymptotic performance of the Root-MUSIC } \\
\text { with smoothing }\left(\Delta \theta_{i}^{2} \text { in } \text { deg }^{2}\right)\end{array}$ \\
\cline { 2 - 3 } & From the algorithm & Evaluation of $(16)$ \\
\hline & $0.4852 \times 10^{-1}$ & $0.4893 \times 10^{-1}$ \\
2 & $0.2929 \times 10^{-1}$ & $0.3105 \times 10^{-1}$ \\
3 & $0.4686 \times 10^{-1}$ & $0.4791 \times 10^{-1}$ \\
4 & $0.2819 \times 10^{-1}$ & $0.2951 \times 10^{-1}$ \\
5 & $0.7611 \times 10^{-2}$ & $0.8719 \times 10^{-2}$ \\
6 & $0.9196 \times 10^{-2}$ & $0.1082 \times 10^{-1}$ \\
7 & $0.2612 \times 10^{-1}$ & $0.2737 \times 10^{-1}$ \\
8 & $0.5241 \times 10^{-2}$ & $0.6161 \times 10^{-2}$ \\
9 & $0.7199 \times 10^{-2}$ & $0.8350 \times 10^{-2}$ \\
10 & $0.8421 \times 10^{-2}$ & $0.9799 \times 10^{-2}$ \\
11 & $0.1081 \times 10^{-1}$ & $0.1257 \times 10^{-1}$ \\
12 & $0.1653 \times 10^{-1}$ & $0.1890 \times 10^{-1}$ \\
\hline
\end{tabular}

an odd multiple of $\pi / 2$, the effective correlation reduces to zero for all $K$. This is not so in the case of ULA.

Equation (13) can be used to study the MSE performance of the Root-MUSIC with forward-backward smoothing applied to the transformed UCA data by replacing the appropriate terms in (13) with the corresponding terms for forward-backward smoothing. Further, (17) can be used for the asymptotic performance for the case of forward-backward smoothing when the sources are uncorrelated.

\section{Spatial Smoothing with Directional Elements}

The analysis carried out so far assumes omnidirectional sensors. The same can be extended to the case of directional sensors. We, however, assume that all the sensors in the UCA are identical. Let $G(\theta)$ be the directional pattern of the elements. For convenience, we assume that the maximum gain of each sensor is along the radial direction. Since the element pattern $G(\theta)$ is a periodic function with period $2 \pi$, it can be expanded in Fourier series [3]. That is

$$
G(\theta)=\sum_{l=-Q}^{Q} c_{l} e^{j l \theta}
$$


TABLE III

Finite Data Performance of the Root-MUSiC with Smoothing Applied to Transformed UCA Data with Directional Elements for Closely Spaced and Correlated Sources $\left(\left[L=50, d=0.4 \lambda, N=100\right.\right.$, DOA's $=0^{\circ}$ AND $\left.5^{\circ}, \rho=0.9 e^{j \pi / 4}, \mathrm{SNR}=6 \mathrm{~dB}, G(\theta)=1+\cos \theta\right]$

\begin{tabular}{|c|c|c|c|c|c|}
\hline \multirow{4}{*}{$\begin{array}{c}\text { Number of } \\
\text { virtual } \\
\text { subarrays } \\
K\end{array}$} & \multicolumn{5}{|c|}{ MSE in DOA estimate $\left(\mathrm{deg}^{2}\right)$} \\
\hline & \multirow{3}{*}{$\begin{array}{l}\text { Spectral MUSIC } \\
\text { applied to } \\
\text { UCA data }\end{array}$} & \multicolumn{4}{|c|}{ Root-MUSIC with smoothing applied to transformed data } \\
\hline & & \multicolumn{2}{|c|}{ Forward-Backward smoothing } & \multicolumn{2}{|c|}{ Forward smoothing } \\
\hline & & Simulation & Evaluation of (13) & Simulation & Evaluation of (13) \\
\hline 1 & 0.02967 & 0.002511 & 0.002930 & 0.01087 & 0.008975 \\
\hline 2 & & 0.002526 & 0.002908 & 0.00931 & 0.009067 \\
\hline 3 & & 0.002694 & 0.003043 & 0.00935 & 0.009312 \\
\hline 4 & & 0.003084 & 0.003366 & 0.01005 & 0.01005 \\
\hline 5 & & 0.003647 & 0.003956 & 0.01177 & 0.01147 \\
\hline 6 & & 0.004245 & 0.004599 & 0.01277 & 0.01290 \\
\hline 7 & & 0.004581 & 0.004902 & 0.01324 & 0.01322 \\
\hline 8 & & 0.004896 & 0.005175 & 0.01317 & 0.01344 \\
\hline 9 & & 0.005338 & 0.005549 & 0.01364 & 0.01396 \\
\hline 10 & & 0.005749 & 0.005920 & 0.01465 & 0.01451 \\
\hline
\end{tabular}

where $Q$ is the number of significant terms in the expansion. If we choose the element directional pattern [3] as $G(\theta)=1+\cos \theta$, then the Fourier coefficients of the pattern are given by $c_{-1}=c_{1}=0.5$, $c_{o}=1$. The steering vector of the UCA with directional elements is now given by

$$
\begin{gathered}
\mathbf{a}_{c d}(\theta)=\left[G(\theta) e^{j \xi \cos \theta}, G\left(\theta-\gamma_{1}\right) e^{j \xi \cos \left(\theta-\gamma_{1}\right)}, \cdots\right. \\
\left.G\left(\theta-\gamma_{L-1}\right) e^{j \xi \cos \left(\theta-\gamma_{L-1}\right)}\right]^{T}
\end{gathered}
$$

where $\gamma_{n}=2 \pi n / L$. If we now apply the weight vector $\mathbf{w}_{m}$, then the resultant array pattern for the $m$ th phase mode is

$$
\begin{aligned}
f_{m}(\theta) & =\mathbf{w}_{m}^{H} \mathbf{a}_{c d}(\theta) \\
& =\frac{j^{-|m|}}{L} \sum_{n=0}^{L-1} G\left(\theta-\gamma_{n}\right) e^{j \xi \cos \left(\theta-\gamma_{n}\right)} e^{j m \gamma_{n}} .
\end{aligned}
$$

Substituting (21) for $G(\theta)$ in (23) and simplifying, we get

$$
\begin{aligned}
f_{m}(\theta)= & \frac{j^{-|m|}}{L} \sum_{l=-Q}^{Q} \sum_{q=-\infty}^{\infty} \\
& \cdot c_{l} e^{j(l-q) \theta} J_{q}(\xi) j^{q} \sum_{n=0}^{L-1} e^{j(q-l+m) \gamma_{n}} .
\end{aligned}
$$

If we now assume that $d<\lambda / 2$, then the resultant array pattern for the $m$ th phase mode can be shown to be

$$
\begin{aligned}
f_{m}(\theta) & =\mathbf{w}_{m}^{H} \mathbf{a}_{c d}(\theta) \\
& =e^{j m \theta} j^{-|m|} \sum_{l=-Q}^{Q} c_{l}\left[j^{l-m} J_{l-m}(\xi)\right]+\Delta f_{m}(\theta)
\end{aligned}
$$

where

$$
\begin{aligned}
\Delta f_{m}(\theta)= & j^{-|m|} \sum_{l=-Q}^{Q} c_{l} \sum_{q=1}^{\infty} \\
& \cdot\left[j^{g+l} J_{g+l}(\xi) e^{-j g \theta}+j^{h-l} J_{h-l}(\xi) e^{j h \theta}\right]
\end{aligned}
$$

with $g$ and $h$ as defined in Section II. Applying the transformation $\mathbf{F}$ to the steering vector, we have $\mathbf{F}^{H} \mathbf{a}_{c d}(\theta)=\mathbf{J}_{d \xi} \mathbf{a}(\theta)+\boldsymbol{\Delta} \mathbf{a}_{d}(\theta)$,
TABLE IV

Finite Data Performance of the Root-MUSiC with Smoothing (APPlicable to Both Forward and Forward-BaCKWARd, Since $\rho$ is Real) Applied to Transformed UCA Data With Directional Elements For Highly CORRELATEd AND Widely SPACED Sources $\left(\left[L=50, N=100\right.\right.$, DOA's $=0^{\circ}$ AND $15^{\circ}$, $d=0.4 \lambda, \mathrm{SNR}=0 \mathrm{~dB}, \rho=0.95, G(\theta)=1+\cos \theta]$

\begin{tabular}{c|c|c|c}
\hline \multirow{2}{*}{$\begin{array}{c}\text { Number of } \\
\text { virtual } \\
\text { subarrays, } K\end{array}$} & \multicolumn{3}{|c}{ MSE in DOA estimate $\left(\mathrm{deg}^{2}\right)$} \\
\cline { 2 - 4 } & $\begin{array}{c}\text { Spectral MUSIC applied } \\
\text { to UCA output }\end{array}$ & \multicolumn{2}{|c}{$\begin{array}{c}\text { Root-MUSIC with smoothing } \\
\text { applied to transformed data }\end{array}$} \\
\cline { 2 - 4 } & & Simulation & Evaluation of $(13)$ \\
\hline 1 & 0.02951 & 0.02433 & 0.02064 \\
2 & & 0.01689 & 0.01578 \\
3 & & 0.01218 & 0.01128 \\
4 & & 0.00890 & 0.00815 \\
5 & & 0.00702 & 0.00625 \\
6 & & 0.00581 & 0.00504 \\
7 & & 0.00494 & 0.00414 \\
8 & & 0.00452 & 0.00366 \\
9 & & 0.00437 & 0.00348 \\
10 & & 0.00432 & 0.00342 \\
11 & & 0.00430 & 0.00339 \\
12 & & 0.00431 & 0.00344 \\
13 & & 0.00421 & 0.00352 \\
14 & & 0.00409 & 0.00359 \\
15 & & 0.00399 & 0.00368 \\
16 & & 0.00393 & 0.00374 \\
17 & & 0.00382 & 0.00376 \\
18 & & 0.00365 & 0.00373 \\
19 & & 0.00352 & 0.00364 \\
20 & & 0.00333 & 0.00348 \\
21 & & 0.00317 & 0.00329 \\
22 & & 0.00308 & 0.00317 \\
23 & & 0.00316 & 0.00313 \\
24 & & 0.00321 & 0.00316 \\
25 & & 0.00325 & 0.00327 \\
26 & & 0.00354 & 0.00350 \\
27 & & 0.00389 & 0.00389 \\
28 & & 0.00447 & 0.00451 \\
\hline & & & \\
\hline & & &
\end{tabular}

where $\mathbf{J}_{d \xi}$ is a diagonal matrix (similar to the matrix $\mathbf{J}_{\xi}$ ) consisting of the elements obtained from the coefficients of $e^{j m \theta}$ in (25), and $\Delta \mathbf{a}_{d}(\theta)$ is the vector consisting of the residual terms in (25). Since the structure of $\mathbf{a}(\theta)$ is similar to that of the steering vector of ULA, we can extend the Root-MUSIC and spatial smoothing to UCA with directional elements subject to the approximations as in the omnidirectional case. Thus, the analysis of Sections III and IV can be extended to this case as well. We should, however, note that $\mathbf{J}_{d \xi}$ 
is complex (unlike $\mathbf{J}_{\xi}$, which is real), and hence, $\mathbf{J}_{d \xi}^{*}$ is to be used wherever appropriate.

\section{Numerical AND Simulation Results}

In the simulations and numerical evaluation, we considered a UCA with identical elements (isotropic or directional). We considered two sources with equal powers in all the simulations. The estimates of the DOA's were obtained by averaging over 100 Monte Carlo runs. The number of snapshots, the number of virtual subarrays, the total array size, and the particulars of signal scenario are described in the captions of figures and tables. The SNR indicated in the figures refers to the value at the input of the sensor element. For spectral MUSIC, the search for DOA's was conducted in steps of $0.002^{\circ}$.

Fig. 1 shows variation of the norm of the effective error vector in the transformed steering vector with smoothing [see (18)]. Note that as the spacing between the elements increases, the norm of the error vector increases and becomes quite large when the spacing approaches $\lambda / 2$. In addition, the norm decreases with smoothing. This behavior is in accordance with our predictions.

To see if the smoothing reduces the effect of the error introduced due to the approximation [c.f. (3)] in the case of both uncorrelated and correlated scenarios, and to evaluate the utility of the theoretical result (17), we applied the Root-MUSIC with forward smoothing to the covariance matrix $\left(\mathbf{R}_{f}^{t}\right)_{w}$. The result so obtained from this is referred to as the asymptotic performance from the algorithm. Tables I and II give this result along with the theoretical values for various values of subarrays. Note from the results of Table I that the MSE is maximum for $K=1$ (no smoothing), and it drops significantly with smoothing. As the smoothing is increased beyond $K=8$, the performance starts deteriorating because of the reduction in the aperture. The results predicted from (17) are not identical to those obtained from the algorithm since the theoretical expressions are accurate for small values of $\Delta \mathbf{a}(\theta)$, which in the present case is not small as $d$ is close to $\lambda / 2$. The small fluctuations in the $\Delta \theta_{i}^{2}$ values with $K$ are because of the fact that the rate at which the norm of the effective error vector [c.f. (18)] and the effective correlation (with smoothing) fall varies with $K$. This explains why the performance for $K=7$ is inferior to that for $K=5$ and 6 . The results for the case of correlated sources are given in Table II. Smoothing improves the performance in this case as well. However, the improvement is not as much as with uncorrelated sources. Since $\rho$ is real, the results of Tables I and II hold for both the forward and forward-backward smoothing.

To demonstrate the impact of smoothing for the case of directional elements with finite data, we considered a closely spaced two-source scenario with $\rho=0.9 e^{j \pi / 4}$. The results are given in Table III. The directional pattern of the elements was chosen as $G(\theta)=$ $1+\cos \theta$. Observe that the performance of the Root-MUSIC with forward-backward smoothing is much better compared with that with forward smoothing only. Further, the forward smoothing alone performs better than the spectral MUSIC applied directly to the UCA data even at higher values of $K$. The performance degradation with increasing value of $K$ is because of the reduction in the aperture, which becomes crucial for closely spaced sources. The simulation results agree well with the theoretically predicted results.

We next considered a widely spaced and highly correlated twosource scenario. The corresponding results are given in Table IV. Since the correlation coefficient between the sources is real, the results hold for both the forward and forward-backward smoothing. Note that the Root-MUSIC with smoothing applied to the transformed UCA data performs much better compared with the spectral MUSIC applied to the UCA data. We also note from results that the MSE is not very sensitive to the number of virtual subarrays, especially for
$8 \leq K \leq 27$. Thus, there exists a wider choice for the number of subarrays to be used gainfully when the sources are widely spaced. Finally, observe that the simulation results agree closely with the predicted ones.

\section{CONCLUSIONS}

The correspondence analyzes the DOA estimation performance of the Root-MUSIC with smoothing applied to the transformed UCA data with isotropic elements and extends the smoothing to UCA with directional elements. It is shown that the smoothing helps in reducing the effect of the errors that arise while extending the RootMUSIC to UCA. This suggests that for a specified performance, the circumferential spacing between the UCA elements can be increased, thereby resulting in larger aperture. It is also shown that in the case of UCA, the forward-backward smoothing reduces to the forward smoothing alone when the source correlations are real.

\section{REFERENCES}

[1] D. E. N. Davies, The Handbook of Antenna Design. London, U.K.: Peter Peregrinus, 1983, vol. 2, ch. 12

[2] C. P. Mathews and M. D. Zoltowski, "Eigenstructure techniques for $2-$ D angle estimation with uniform circular arrays," IEEE Trans. Signal Processing, vol. 42, pp. 2395-2407, Sept. 1994.

[3] T. Rahim and D. E. N. Davies, "Effect of directional elements on the directional response of circular antenna arrays," Proc. Inst. Elect. Eng. H, vol. 129, pp. 18-22, Feb. 1982.

[4] B. D. Rao and K. V. S. Hari, "Effect of spatial smoothing on the performance of MUSIC and minimum-norm method," Proc. Inst. Elect. Eng. F, vol. 137, pp. 449-458, Dec. 1990.

[5] V. U. Reddy, A. Paulraj, and T. Kailath, "Performance analysis of the optimum beamformer in the presence of correlated sources and its behavior under spatial smoothing," IEEE Trans. Acoust., Speech, Signal Processing, vol. ASSP-35, pp. 927-936, July 1987.

[6] K. M. Reddy and V. U. Reddy, "Analysis of interpolated arrays with spatial smoothing," Signal Process., vol. 54, pp. 261-272, Nov. 1996.

[7] T. J. Shan, M. Wax, and T. Kailath, "On spatial smoothing for directions of arrival estimation of coherent signals," IEEE Trans. Acoust., Speech, Signal Processing, vol. ASSP-33, pp. 806-811, Aug. 1985.

[8] A. H. Tewfik and W. Hong, "On the application of uniform linear array bearing estimation techniques to uniform circular arrays," IEEE Trans. Signal Processing, vol. 40, pp. 1008-1011, Apr. 1992.

[9] R. T. Williams, S. Prasad, A. K. Mahalanabis, and L. H. Sibul, "An improved spatial smoothing technique for bearing estimation in a multipath environment," IEEE Trans. Acoust., Speech, Signal Processing, vol. 36, pp. 425-431, Apr. 1988.

[10] M. Wax and J. Sheinvald, "Direction finding of coherent signals via spatial smoothing for uniform circular arrays," IEEE Trans. Antennas Propagat., vol. 42, pp. 613-620, May 1994.

[11] R. Eiges and H. D. Griffiths, "Mode space spatial spectral estimation for circular arrays," Proc. Inst. Elect. Eng. F, Radar, Sonar, Navigat., vol. 141, pp. 300-306, Dec. 1994. 